Europhysics Letters

PREPRINT

\title{
Loading of a Bose-Einstein condensate in the boson-accumulation regime
}

\author{
F. Floegel, L. Santos And M. Lewenstein \\ Institut für Theoretische Physik, Universität Hannover, D-30167 Hannover, Germany
}

\begin{abstract}
PACS. 03.75.Fi - Phase coherent atomic ensembles; quantum condensation phenomena. PACS. 42.50.Vk - Mechanical effects of light on atoms.

PACS. 32.80.-t - Photon interactions with atoms.
\end{abstract}

\begin{abstract}
We study the optical loading of a trapped Bose-Einstein condensate by spontaneous emission of atoms in excited electronic state in the Boson-Accumulation Regime. We generalize the previous simplified analysis of ref. [Phys. Rev. A 53, 2466 (1996)], to a 3D case in which more than one trap level of the excited state trap is considered. By solving the corresponding quantum many-body master equation, we demonstrate that also for this general situation the photon reabsorption can help to increase the condensate fraction. Such effect could be employed to realize a continuous atom laser, and to overcome condensate losses.
\end{abstract}

During the last years the experimental realization of Bose-Einstein condensation (BEC) in trapped weakly interacting gases [1] has stimulated an enormous interest [2]. Among the results related to BEC, specially remarkable is the realization of an Atom Laser [3 6. As a coherent source of matter waves, the atom laser will lead to new applications in atom optics. Several groups have demonstrated atom lasers using pulsed [3, 6] or continous [5] outcoupling from the BEC, either by employing rf fields [3], or Raman pulses [6]. However, the continuous outcoupling is just a half way towards a $\mathrm{cw}$ atom laser. The continuous loading of the condensate still remains to be realized. Like in the developement of light lasers the availability of $\mathrm{cw}$ atom lasers would open the way to "high power" and precision applications.

Two different physical mechanisms could provide continuous pumping of atoms into a condensate. On one hand, this can be achieved by collisional mechanisms [7], in which two non-condensed atoms collide, one being pumped into the condensate, whereas the other being evaporated. On the other hand, the optical pumping into a BEC via spontaneous emission has also been proposed [8]. If the reservoir could be filled in a (quasi-) continuous way by laser cooling techniques, one would benefit from the large cooling efficiency of laser cooling compared to evaporative cooling, allowing for a considerable increase in atomic flux produced by an atom laser. For the latter, it is crucial that the spontaneously emitted photons cannot be reabsorbed, since otherwise heating is introduced in the system, and BEC can be neither achieved, nor maintained [9. It has been shown that in the so-called Festina Lente regime, in which the spontaneous emission rate $\gamma$ is smaller than the trap frequency $\omega$, the heating due to the reabsorption processes is suppressed $[10]$. However, due to the slow time constants in this approach the cooling efficiency is greatly reduced. Recently, it has been also reported [11]

(C) EDP Sciences 
that when an atom possesses an accessible three level $\Lambda$ scheme, where one of the atomic transitions decays much faster than the other, the reabsorptions in the slow transition can be largely suppressed, without the time limitations of Festina Lente.

In this Letter we study the loading of a BEC, which is formed in a trapped electronic ground-state $|g\rangle$, via spontaneous decay of atoms from an also trapped internal excited state $|e\rangle$. In the regime $N_{0} \gg a, N-N_{0}$ (where $a$ is the effective number of levels other than the condensed one to which the excited atoms may decay, $N$ is the total number of atoms in the $|g\rangle$ trap, and $N_{0}$ is the number of condensed ones) the atom will decay into the condensate with high probability, due to the bosonic enhancement. Such regime has been named BosonAccumulation Regime (BAR) [12]. Note, however, that even in the BAR, in the absense of reabsorption simple arguments imply that the mean proportion of atoms in the condensate after the decay decreases [12]. If the reabsorptions are present, each subsequent decay would lead to an even larger decrease of the condensate proportion; thus for an optically thick sample (where many reabsorptions take place) the number of condensed atoms would be reduced dramatically. However, these arguments are not rigorously valid in general. In ref. [12] it was reported that a fully quantum treatment shows that the reabsorption processes can, under certain conditions, help to increase the proportion of atoms in the BEC. This counterintuitive result was explained by means of an interference effect between different paths (which include reabsorptions) that lead to the same final states. This effect offers an interesting mechanism of continuous refilling and loading of a condensate.

The treatment of ref. 12] was based on an extremely simplified 1D model in which all the ground states were taken into account, but in which there was a single excited level. In addition, the calculations of ref. 12 did not consider the different Frank-Condon factors for the different possible decays. In this Letter, we analyze the BAR regime in a much more general model. We consider a 3D trap, and the possibility of more than one excited trap level. In addition, the Frank-Condon factors for the different decays are explicitely included in the calculations. We demonstrate that even for this much more complicated situation, under some conditions the reabsorption still helps to load atoms into the condensate.

Let us consider a set of bosonic atoms with two internal levels $|g\rangle$ and $|e\rangle$ confined in a dipole harmonic trap, which for simplicity is considered isotropic, with frequency $\omega$. We denote as $N_{m}$ the population of the $m$-th level of the $|g\rangle$ trap, where $m \equiv\left(m_{x}, m_{y}, m_{z}\right)$, and assume that the ground state trap verifies the BAR conditions. We analyze the situation in which a single atom in some state $|e, l\rangle\left(l \equiv\left(l_{x}, l_{y}, l_{z}\right)\right)$ decays via spontaneous emission into the $|g\rangle$ trap, producing a photon which can eventually be reabsorbed by another $|g\rangle$ atom (in particular by a condensed one), which in turn can later decay again, and so on. At some finite time, the scattered photon is no more reabsorbed and leaves the system. It is our aim to investigate how the atom distribution in the $|g\rangle$ trap changes during this process.

For simplicity we do not consider the collisional mean-field effects. For typical $s$-wave scattering lengths this implies that in principle our results could be obtained for few atoms only (since we consider relatively small traps); one should point out, however, that although our calculations are limited to small traps, the BAR effect should be present also for larger ones, and in the presence of collisions. Although the main purpose of this Letter is to discuss a fundamental quantum effect and to present the methodology needed to study it, we must also point out that recent experiments 13 have shown that the $s$-wave scattering length can be modified by using Feshbach resonances. In particular, the regime of quasi-ideal gas can be experimentally achieved, in which the mean-field energy can be considered smaller than the trap energy. In such regime, the collisions introduce just a thermalization mechanism [14], and two and three-body collisional losses are almost absent [15]. For such modified scattering length our results are valid for much larger $N$. 
Starting from the Hamiltonian which describes the bosons interacting with the quantized electromagnetic field, and using standard techniques, one can derive the master equation (ME) for the reduced density operator for the atomic degrees of freedom [16], which assuming $\hbar=1$ becomes

$$
\dot{\rho}=-i H_{e f f} \rho+i \rho H_{e f f}^{\dagger}+J \rho
$$

where

$$
H_{e f f}=\sum_{l=0}^{\infty}\left(\omega_{l}^{e}+\omega_{0}-i \frac{\Gamma}{2}\right) e_{l}^{\dagger} e_{l}+\sum_{k=0}^{\infty} \omega_{k}^{g} g_{k}^{\dagger} g_{k}-i \frac{\Gamma}{2} \sum_{l, l^{\prime}}^{\infty} \sum_{m, m^{\prime}}^{\infty} \alpha_{l m m^{\prime} l^{\prime}} g_{m^{\prime}}^{\dagger} g_{m} e_{l}^{\dagger} e_{l^{\prime}}
$$

is a non-hermitian effective Hamiltonian, and

$$
J \rho=\Gamma \sum_{l, l^{\prime}}^{\infty} \sum_{m, m^{\prime}}^{\infty} \alpha_{l m m^{\prime} l^{\prime}}^{r} g_{m^{\prime}}^{\dagger} e_{l^{\prime}} \rho e_{l}^{\dagger} g_{m} .
$$

is the jump operator. Here, $e_{l}\left(g_{m}\right)$ is the annihilation operator for atoms in the $l-$ th $(m-$ th) excited (ground) level, $\omega_{l}^{e}\left(\omega_{m}^{g}\right)$ is the energy corresponding to such state, and $\Gamma$ is the spontaneous emission rate from the excited level. The complex coefficients $\alpha_{l m m^{\prime} l^{\prime}}$ (whose explicit form can be found in [12]) are related to the Frank-Condon factors for the different excited-ground transitions. We denote in the following $\eta_{l m}$ the Franck-Condon factor for the transition between the $l$-th excited level and the $m$-th ground level. The real part of the $\alpha_{l m m^{\prime} l^{\prime}}$ coefficient is denoted $\alpha_{l m m^{\prime} l^{\prime}}^{r}$. We consider an initial situation in which $N_{m}$ atoms occupy the $m$-th state of the ground-state trap, and a single excited atom is placed (with some probability given by a thermal distribution) in a state $j$ of its corresponding trap. We denote the initial state as $\left|\psi_{0}\right\rangle$, and therefore the initial density matrix is defined as $\rho_{N}=\left|\psi_{0}\right\rangle\left\langle\psi_{0}\right|$. After a photon is released without further reabsorptions, we obtain the formal solution $\rho_{N+1}$ of the ME (1), and calculate the probability to obtain a particular final state $\left|\psi_{f}\right\rangle$ with $N_{m}^{\prime}$ atoms in the $m$-th $|g\rangle$ trap level (and no atom in the excited state trap). After expanding into powers of the small parameter $N_{0}^{-1 / 2}$, and neglecting terms of order $\mathcal{O}\left(N_{0}^{-3 / 2}\right)$, such probability takes the form

$$
\left\langle\psi_{f}\left|\rho_{N+1}\right| \psi_{f}\right\rangle=\frac{2}{N_{0}} \int_{0}^{\infty} d t \int \frac{d \Omega}{4 \pi}\left|\sum_{l} \eta_{l 0}^{*}\right|\left\langle\psi_{f}\left|g_{0}^{\dagger} e_{l} A_{1}\right| \psi_{0}\right\rangle+\left.\sum_{l, m \neq 0} \eta_{l m}^{*}\left\langle\psi_{f}\left|g_{m}^{\dagger} e_{l} A_{0}\right| \psi_{0}\right\rangle\right|^{2}(4)
$$

with $A_{0}(t)=\exp \left[-i H_{e f f}^{(0)} t\right]$ and $A_{1}=-i \int_{0}^{t} d \tau \exp \left[-i H_{e f f}^{(0)}(t-\tau)\right] H_{e f f}^{(1)} \exp \left[-i H_{e f f}^{(0)} \tau\right]$ being the terms of $\exp \left[-i H_{e f f} t\right]$ of order 0 and 1 in $1 / N_{0}^{1 / 2}$, where

$$
H_{e f f}^{(0)}=\omega_{0}^{g} g_{0}^{\dagger} g_{0}-i \frac{\Gamma}{2} \sum_{l, l^{\prime}}^{\infty} \alpha_{l 00^{\prime} l^{\prime}} g_{0}^{\dagger} g_{0} e_{l}^{\dagger} e_{l^{\prime}}
$$

and

$$
H_{e f f}^{(1)}=-i \frac{\Gamma}{2} \sum_{l, l^{\prime}}^{\infty} \sum_{m \neq 0}^{\infty}\left\{\alpha_{l m 0 l^{\prime}} g_{0}^{\dagger} g_{m}+\alpha_{l 0 m l^{\prime}} g_{m}^{\dagger} g_{0}\right\} e_{l}^{\dagger} e_{l^{\prime}}
$$

are respectively the terms of $H_{\text {eff }}$ of zeroth and first order in $1 / N_{0}^{1 / 2}$. Therefore, beginning from an initial relative number of condensate particles $n=N_{0} / N$, up to order $\mathcal{O}\left(1 / N_{0}^{2}\right)$, the 
new relative number of condensed atoms after the last decay becomes

$$
n^{\prime}=\frac{N_{0}+1+\left(P_{N_{0}+2}-P_{N_{0}}\right)}{N+1}
$$

where $P_{N_{0}+2}\left(P_{N_{0}}\right)$ is the probability to have after the process $N_{0}^{\prime}=N_{0}+2\left(N_{0}\right)$. Let $\left|\psi_{f}^{(2, s)}\right\rangle$ be the final state with $N_{0}^{\prime}=N_{0}+2, N_{s}^{\prime}=N_{s}-1$, and $N_{j \neq 0,2}^{\prime}=N_{j}$. The probability to decay into such a state is given by:

$$
P_{N_{0}+2}^{s}=\left\langle\psi_{f}^{(2, s)}\left|\rho_{N+1}\right| \psi_{f}^{(2, s)}\right\rangle=\frac{2}{N_{0}} \int_{0}^{\infty} d t \int \frac{\Omega}{4 \pi}\left|\sum_{l, m \neq 0} \eta_{l m}^{*}\left\langle\psi_{f}^{(2, s)}\left|g_{m}^{\dagger} e_{l} A_{0}(t)\right| \psi_{0}\right\rangle\right|^{2} .
$$

The corresponding process is schematically represented in fig. 11(a). Let us also define $\left|\psi_{f}^{(0, s)}\right\rangle$ as the final state with $N_{0}^{\prime}=N_{0}, N_{s}^{\prime}=N_{s}+1$ and $N_{j \neq 0,2}^{\prime}=N_{j}$. The probability to decay into this state takes the form:

$$
\begin{aligned}
P_{N_{0}}^{s}= & \left\langle\psi_{f}^{(0, s)}\left|\rho_{N+1}\right| \psi_{f}^{(0, s)}\right\rangle \\
= & \frac{2}{N_{0}} \int_{0}^{\infty} d t \int \frac{\Omega}{4 \pi}\left\{\left|\sum_{l} \eta_{l 0}^{*}\left\langle\psi_{f}^{(0, s)}\left|g_{0}^{\dagger} e_{l} A_{1}(t)\right| \psi_{0}\right\rangle\right|^{2}+\left|\sum_{l, m \neq 0} \eta_{l m}^{*}\left\langle\psi_{f}^{(0, s)}\left|g_{m}^{\dagger} e_{l} A_{0}(t)\right| \psi_{0}\right\rangle\right|^{2}\right. \\
& \left.+2 \Re\left\{\sum_{l} \eta_{l 0}^{*} \mid\left\langle\psi_{f}^{(0, s)}\left|g_{0}^{\dagger} e_{l} A_{1}(t)\right| \psi_{0}\right\rangle\left(\sum_{l, m \neq 0} \eta_{l m}^{*}\left\langle\psi_{f}^{(0, s)}\left|g_{m}^{\dagger} e_{l} A_{0}(t)\right| \psi_{0}\right\rangle\right)\right\}\right\}
\end{aligned}
$$

where $\Re$ denotes the real part. The first and second terms in eq. (9) are respectively depicted in fig. 1 (b) and (c), whereas the last term corresponds to the intereference between the energetically equivalent paths which are shown in that figures. From eqs. (8), (9) one obtains $P_{N_{0}+2}=\sum_{s}<P_{N_{0}+2}^{s}>$, and $P_{N_{0}}=\sum_{s}<P_{N_{0}}^{s}>$, where $<>$ denotes the average of the possible initial ground-state populations following the corresponding Bose-Einstein distribution. We have numerically calculated the probabilities $P_{N_{0}}$ and $P_{N_{0}+2}$. From eqs. (8), (9) it becomes clear that such calculation requires to take into account all the possible paths connecting a particular initial and final state which eventually involves an arbitrary number of emission-reabsorption cycles. This fact by itself makes the calculation extremely demanding. In addition, several important technical difficulties appear. First, we must note that in order to calculate the coefficients $A_{0}$ and $A_{1}$, one has to evaluate the exponential $\exp (-i \hat{M} t)$, where $\hat{M}=\sum_{l, l^{\prime}} \alpha_{l 00 l^{\prime}} e_{l}^{\dagger} e_{l^{\prime}}$ is not an Hermitian matrix. Therefore, it becomes neccesary to introduce a biorthogonal set of operators $f_{R}^{\dagger}(k)=\sum_{l} v_{l}^{R}(k) e_{l}^{\dagger}$, and $f_{L}(k)=\sum_{l} \bar{v}_{l}^{L}(k) e_{l}$, where $v_{l}^{R}(k)$ and $\bar{v}_{l}^{L}(k)$ are the right and the left (complex conjugate) eigenvectors of $\hat{M}$. Therefore, such eigenvectors, and eigenvalues have to be calculated. Second, we note that the $\alpha$ coefficients constitute a 12 dimensional tensor, and that in order to evaluate every component, it is neccesary to calculate the Franck-Condon factors for each possible excited-ground transition, and to perform the non-trivial task of calculating the Cauchy principal part integral appearing in the imaginary part of the $\alpha$ coefficients [12]. Such strong technical difficulties make eventually impossible to solve in $3 \mathrm{D}$ in a reasonable computational time systems with more than 4 excited-state shells (20 levels), and therefore we have been constrained by such limit.

We have analyzed the case of different number of energy shells of the $|e\rangle$ and $|g\rangle$ trap, for different temperatures and different number of atoms. For each case, we have calculated 


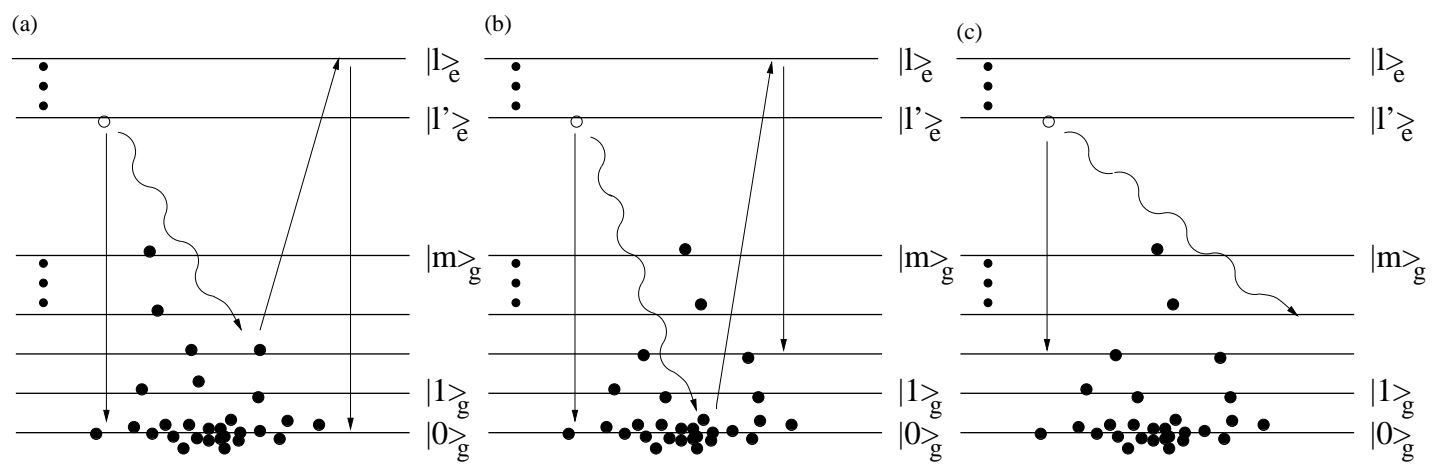

Fig. 1 - Schematic representation of the different processes appearing in eqs. (8) and (9). Straight lines denote atomic transitions, whereas waved lines represent the photons produced in the decays. Note the possibility of reabsorption in figs. (a) and (b).

the probabilites $(80,(9)$, in order to evaluate the new relative number of condensed atoms $n^{\prime}$. For the particular case of a single level in the excited state trap, results similar to those obtained in ref. [12] were obtained. Figure 2 shows for different temperatures of the excited and ground trap, the case of 4 shells (20 levels) in the $|e\rangle$ trap, 10 shells (220 levels) in the $|g\rangle$ trap, $N=7 \times 10^{4}$ atoms, and a Lamb-Dicke parameter $\eta^{2}=\omega_{r} / \omega=2$, where $\omega_{r}$ is the recoil energy of the scattered photon. One can observe that for certain temperatures $T_{e}$ and $T_{g}$ of the $|e\rangle$ and $|g\rangle$ traps, the change in the relative number of condensed particles, $n^{\prime}-n$ is maximally positive, i.e. at these maxima the reabsorption effects help in the most efficient way to load the condensate. Away from such maxima, $n^{\prime}-n$ is positive for low temperature $T_{e}$ of the excited state trap and not very low temperatures $T_{g}$ of the $|g\rangle$. For very low $T_{g}$, however, the positive processes described by $P_{N 0+2}$ tend to vanish, since the number of non-condensed atoms which eventually could be repumped to the condensate becomes very small. On the top of fig. 2, such regions are those enclosed by the contours. Out of these regions, $n^{\prime}-n$ becomes negative, i.e. the reabsorption tends to decrease the condensation relative number. In all our calculations the BAR expansion has been proved to be valid, by checking the condition $P_{N_{0}}, P_{N_{0}+2} \ll 1$. We have in general observed that the BAR can fail not only for large $T_{g}$, as expected from ref. [12], but eventually also, for a given total number of atoms $N$, for large $T_{e}$ at the $n^{\prime}-n$ maxima. The latter is due to the large values of the imaginary part of the $\alpha$ coefficients at those peaks under such conditions, which invalid the expansion performed to obtain eqs. (8) and (9). In the presented example, the condition above is fulfilled except for the maxima in the region $k_{B} T_{e}>\omega$ and $k_{B} T_{g}>50 \omega$. As discussed in ref. [12], the phenomenon behind the positive effects of the reabsorption $\left(n^{\prime}-n>0\right)$ cannot be explained by using (classical) rate equations, since it is given by the interference between the different paths which lead to the same final state. In particular, it becomes decisive that the interference term in eq. (9) is always destructive, since the process of fig. 2(b) includes an additional absorption-emission cycle (which gives a minus sign in the amplitude, like for a $2 \pi$-laser pulse). The consequence of this interference is that $P_{N_{0}}$ decreases, which favours that the excited atom goes to the state $\left|N_{0}+1\right\rangle$, and therefore it contributes to an increase of the proportion of condensed atoms.

We have numerically simulated the pumping of atoms into a BEC in the $|g\rangle$ trap, under the BAR conditions. In our calculations, we have assumed that the collisions act on a shorter time scale than the spontaneous optical pumping. In that case, collisions will provide a fast 
EUROPHYSICS LETTERS

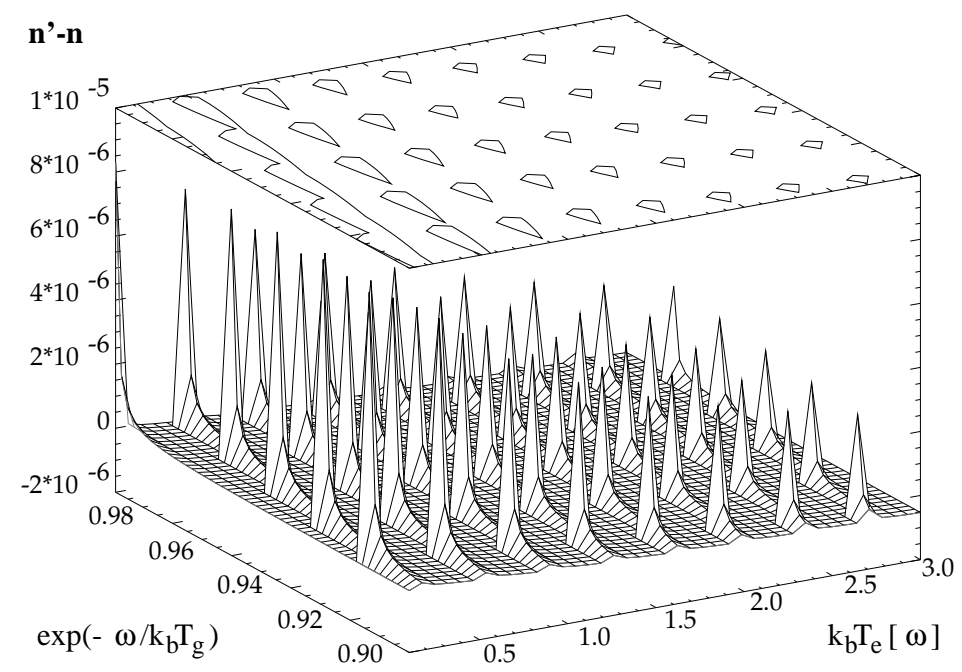

Fig. 2 - Change of the condensate fraction after a decay process as a function of the temperature $T_{e}$ of the $|e\rangle$-trap and $T_{g}$ of the $|g\rangle$-trap, the latter in exponential scale. The case of a total number of atoms $N=7 \times 10^{4}, 10$ energy shells in the $|g\rangle$-trap, 4 energy shells in the $|e\rangle$-trap and $\eta^{2}=2$ is considered. On the top of the figure, the regions enclosed by the contours denote those temperatures for which the condensate fraction increases during the decay process

thermalization mechanism of the $|g\rangle$ trap, between the two pump acts. After each pumping process we calculate the new condensate fraction, and accordingly the new $T_{g}$, which we employ to evaluate the next pumping, and so on. In fig. 3 we analyze the same trap as in fig. 2, for $k_{B} T_{e}=\omega$, and an initial number of trapped atoms $N=5 \times 10^{4}$, for initial $N_{0} / N=0.9$, 0.98 and 0.99. One can observe that the positive effects of the reabsorption in the BAR regime allow to increase the condensate fraction during the pumping process, up to almost complete condensation. However, as pointed out previously, for very low $T_{g}$ the positive effects of BAR vanish. This explains the fact that the $N_{0} / N$ is slightly lower than 1 , and also that for the case of initial $N_{0} / N=0.99$ the condensate fraction initially decreases.

In this Letter we have extended the results presented in ref. 12] for a much more general $3 \mathrm{D}$ situation, in which the excited state atoms can occupy more than one trapped state. We have shown that under the appropriate conditions, the BAR expansion is still valid for this more general case, and that the reabsorptions can play a positive role in the loading of the condensate. Such effect is a consequence of the always destructive interference between the processes which tend to lower the condensate fraction. The generalized treatment shows that the BAR condition presented in [12] is not enough to guarantee the BAR expansion, since for more than one excited-trap level, the temperature of such trap is also important, and must be kept sufficiently low. Although for complexity reasons we have not analysed the situation in which the trap levels are distorted by the mean field provided by the atom-atom collisions, we must stress that similar analysis could be applied also if the atom-atom collisions modify the levels, if instead of the bare trap levels, self-consistent levels were considered, as in ref. [17]. Therefore, under the BAR conditions, i.e. large condensation, the reabsorption processes favour the optical pumping of atoms via spontaneous emission into a BEC. Such quantum effect is of fundamental interest, but can also be important experimentally to overcome condensate losses, and to achieve of a continuously-loaded atom laser. 


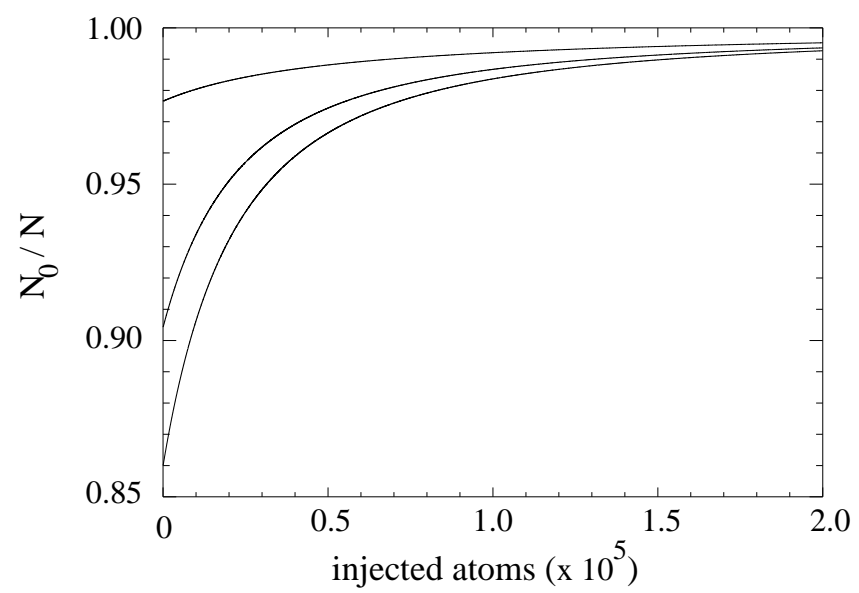

Fig. 3 - Mean condensate fraction as a function of the number of loaded atoms, for the case of 10 energy shells in the $|g\rangle$-trap, 4 energy shells in the $|e\rangle$-trap and $\eta^{2}=2$, and an initial total number of atoms $N=5 \times 10^{4}$. From top to bottom, the curves represent, respectively, the case of an initial number of condensed particles $N_{0}=49632,48828$ and 45211.

We acknowledge support from Deutsche Forschungsgemeinschaft (SFB 407), from the EU through the TMR network ERBXTCT96-0002, and from ESF PESC Programm BEC2000+, and discussions with M. Kottke, K. Sengstock and W. Ertmer.

\section{REFERENCES}

[1] Anderson M. H. et al., Science, 269 (1995) 198; Davis K. B. et al., Phys. Rev. Lett., 75 (1995) 3969; BRAdLEy C. C. et al., ibid, 78 (1997) 985.

[2] Inguscio M., Stringari S. and Wieman C. (Editors), Proceedings of the International School of Physics "Enrico Fermi", Vol. Course CXL, (IOS Press, Amsterdam) 1999.

[3] Andrews M. R. et al., Science, 275 (1997) 637.

[4] Anderson B. P. and Kasevich M.A., Science, 282 (1998) 1686.

[5] Bloch I., Hänsch T.W. And Esslinger T., Phys. Rev. Lett., 82 (1999) 3008.

[6] Hagley E. W. et al., Science, 283 (1999) 1706.

[7] Mandonnet E. et al., Euro. Phys. J. D, 10 (1999) 9.

[8] Spreeuw R. J. C. et al., Europhys. Lett., 32 (1995) 469; Janicke U. and Wilkens M., Adv. At. Mol. Opt. Phys., 41 (1999) 261.

[9] Olshan'in M., Castin Y. and Dalibard J., Proc. 12 ${ }^{\text {th }}$ Int. Conf. on Laser Spectroscopy, edited by M. Inguscio, M. Allegrini, and A. Lasso (World Scientific, Singapour) 1996

[10] Cirac J. I., Lewenstein M. and Zoller P., Europhys. Lett., 35 (1996) 647.

[11] Santos L. et al., , cond-mat/0007003.

[12] Cirac. J. I. and Lewenstein M., Phys. Rev. A, 53 (1996) 2466.

[13] Stenger J. et al., Phys. Rev. Lett., 82 (1999) 2422; Cornish S. L. et al., ibid, 85 (2000) 1795.

[14] Santos L. and Lewenstein M., Appl. Phys. B, 69 (1999) 363.

[15] Roberts J. L. et al., Phys. Rev. Lett., 85 (2000) 728.

[16] Gardiner C. and Zoller P., Phys. Rev. A, 55 (1997) 2902. Jaksch D. et al., Phys. Rev. A, 56 (1997) 575.

[17] Santos L. et al., J. Phys. B, 33 (2000) 4131. 